\title{
A Study of Financial Distress Prediction based on Discernibility Matrix and ANN
}

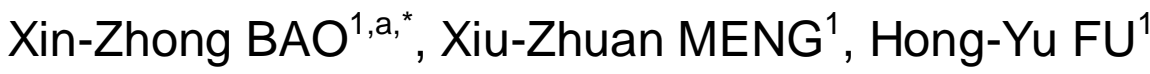 \\ ${ }^{1}$ School of management, Beijing Union University, Beijing, China \\ axinzhongbao@vip.sina.com \\ ${ }^{*}$ Corresponding author
}

Keywords: Rough set, Artificial neural network, Financial distress prediction.

\begin{abstract}
Financial distress prediction is a significant issue that concerns stakeholders of enterprises. Due to the limitation of the samples available, most studies of financial prediction generally catagorize the financial situations of companies by whether they are bankrupt or specially treated in the stock market. In addition, the variables for prediction are determined mainly by subjective judgments. In order to overcome the influence of these limitations, this paper establishes a reduced variable system with sufficient information by rough set discernibility matrix method, and forms a progressive classification of financial situations by hierarchical clustering. These classifications are transformed as the target value of artificial neural network output layer to enhance the interpretation ability of the whole network. Combined with the inputs determined by Rough Set reduction, a neural network model is established to predict the financial distress, especially the turning point of financial degeneration.
\end{abstract}

\section{Introduction}

Because of the profound practical significance of financial distress prediction, research is carried out widely in various statistical methods. Since 2000, data mining and artificial intelligence were introduced to the financial distress prediction for the non-linear and distributed computing power. Lee and Booth et al. (2005) proved the superiority of supervised neural networks by comparing the accuracy of the BP neural network and Konhonen self-organizing feature map neural network in bankruptcy prediction. Min and Lee et al. (2006) used the combination of genetic algorithms and support vector machines (SVM) to predict bankruptcy, which improved the performance of SVM from the aspects of feature subset selection and parameter tuning. Cho and Kim (2009) optimized the binary classification of financial prediction with the consideration of multiple discriminate analysis, logistic regression, decision tress and neural networks. Javier and Pedro (2011) constructed a prediction model based on the fuzzy C-means clustering and multiple adaptive regressions, which was superior to the discriminate analysis and feed forward neural networks tested by the empirical data. Premachandra and Watson (2011) proposed a method to evaluate the success index of an enterprise using data envelopment analysis (DEA) method.

According to the previous literature, two deficiencies exist. First, the financial situation is generally divided into two types of healthy and bankrupt, which obviously have some limitations. Second, the prediction indicators are usually selected subjectively. According to these limitations, this paper will take these following points into consideration.

(1) The classification of financial situation. This paper structures the progressive classification levels of financial situation to improve the traditional division of healthy and bankrupt.

(2) Choosing the right financial indicators. Form the multi-attributes decision table of financial situation level and financial indicators to make further reduction using different matrix.

(3) Building the neural network for financial distress prediction. According to the new decision table, take the number of condition attributes as neural network input layer nodes, the number of decision attributes category as the output layer nodes and build the neural network. 


\section{Research method and process design}

In this paper, we applied rough set theory for data pre-processing and neural network for black-box simulation. The use of rough set theory could extract characteristics from the data itself and winkle the redundant information while maintaining the classification ability. Based on this preprocessing information, the complexity of data input and difficulty of training in follow-up neural network model are highly decreased. Therefore, the forecast accuracy and reasonableness will be greatly enhanced. This paper will complete the construction of financial prediction model by the following process.

Step 1 Determining the primary index and sample

Financial indicators in source of annual reports are selected to avoid the distortion of subjectively quantized non-financial indicators. In addition, this paper selects manufacturing industry companies listed on the Shanghai Stock Exchange as sample. Besides, those companies of B-share or H-share are winkled.

As for the year of data selection, the annual reports of manufacturing industry in year 2010 are selected as sample and the financial situation of year 2012 as the test sample.

Step 2 Data preprocessing

This paper takes deviation standardization to transform the variable value ranged in $[0,1]$ and of pure quantity. As for the standardization process, each financial indicator has its own meaning and average level, thus we determine the maxim and minimum value of each indicator in accordance with relevant research and analysis to go ahead with standardization. This treatment makes the data distributed between 0 and 1 , and eliminates the individual deviation far from the mean value. Accordingly, a condition attributes of decision table is constructed by ROSETTA processing.

Step 3 Cluster analysis

This paper uses hierarchical clustering analysis to form a gradually changed financial situation classification. And in accordance with the situation level, each is valued with 0 or 1 to form the decision attributes of decision table.

Step 4 Attribute reductions using rough set theory

Input the decision table mentioned above into the discernibility matrix generator produced by $\mathrm{C}++$ programming in order to receive the equivalence classes and difference matrix. Through the ways of algorithm mentioned, the attributes reduction set is gained.

Step 5 Neural network training

After a rough set reduction, we take the attributes number as input layer nodes and the clustering financial situation number as the output layer nodes to build a neural network topology optimization model and ultimately form the early warning model.

\section{Empirical study of financial distress prediction}

\section{Determine the primary index}

Based on the method and process discussed above, we take the primary indicator selection as the first step. According to the relative theories and previous studies, this paper considers the capacity of solvency, operation, growth and profitability to form the primary indicator system which includes 15 indicators for the further study.

\section{Data preprocessing}

According to the indicators above and the annual data of financial report, data are prepared for the standardized deviation via practical significance of indicators within the distribution and industrial characteristics.

\section{Cluster analysis}

SPSS software is used to carry out the hierarchical clustering analysis according to company code. We take significance test for the clustering results and analyze the difference between groups to determine the appropriate classification level. According to the analysis report, the sample companies are divided into five classes which have a significant difference between groups. 
The financial situations of sample companies are divided into five classes which are named healthy, good, moderate, inferior and warning.

After finishing the classification arrangement, this paper takes financial data of the sample companies in year 2012 to test the results. 23 companies are special treated in 2012, among which 10 companies located in warning level, 8 located in inferior level while 5 located in moderate. Generally speaking, $78.3 \%$ ST companies are located in the distress level of inferior and warning, while $21.7 \%$ locate in moderate level. Combined with the ANOVA report, the sample classification is tested to be reasonable which could provide a well objective basis for the follow-up neural network study.

\section{Attributes reduction using $\mathrm{RS}$}

We build a decision-making table which contains decision attributes formed by clustering and condition attributes formed by ROSETTA reduction, where code refers to the company code, and values of $\mathrm{D}$ from 0 to 4 refer to the financial situation from warning to healthy.

Take difference matrix analysis for the coordination decision-making table, a reduced decision-making table is formed. According to the attribute significance a new index system is built as table 1 shown.

Table 1 Index system after reduction

\begin{tabular}{c|c|c|c}
\hline Indicator Property & Indicator Name & Mark & Attribute Significance \\
\hline $\begin{array}{c}\text { Short term } \\
\text { Solvency }\end{array}$ & current ratio & $C_{1}$ & 0.1812 \\
\cline { 2 - 4 } & asset-liability ratio & $C_{2}$ & 0.2528 \\
\hline $\begin{array}{c}\text { Long term } \\
\text { Solvency }\end{array}$ & cash-current liability ratio & $C_{3}$ & 0.2013 \\
\hline \multirow{2}{*}{$\begin{array}{c}\text { Operating } \\
\text { Capacity }\end{array}$} & inventory turnover & $C_{4}$ & 0.2819 \\
\cline { 2 - 4 } & $\begin{array}{c}\text { accounts receivable } \\
\text { turnover }\end{array}$ & $C_{5}$ & 0.1185 \\
\hline \multirow{2}{*}{ Profitability } & return on equity & $C_{6}$ & 0.2125 \\
\cline { 2 - 4 } & return on assets & $C_{7}$ & 0.2125 \\
\hline \multirow{2}{*}{ Growth capacity } & revenue growth & $C_{8}$ & 0.0246 \\
\cline { 2 - 4 } & operating profit growth & $C_{9}$ & 0.4698 \\
\hline
\end{tabular}

\section{ANN training}

Based on the analysis above, a three-layer BP neural network is then constructed. The input layer is a buffer memory for receiving external data. Thus the nodes number of input layer is determined by the actual problem. In this paper, it is the indicator number after rough set reduction to form the input layer. As for the output layer nodes, usually they are determined by the types of data and methods used. And in this paper, the classification number to be determined is selected to be the total number of output layer, which is 5 here. In BP network design, the hidden layer nodes will directly affect the performance of neural network. As a network with multiple hidden layers does not help to improve the accuracy of training, this paper set up a network with one hidden layer. The number of nodes in the hidden layer is determined according to equation 3-1: $L=\sqrt{I+O}+\alpha$, where $I=9, O=5, \alpha=6.3$. Thus a reasonable network hidden layer nodes number is $L=10$.

Another factor affect the efficiency of neural network training is transfer function. This paper uses hyperbolic tangent transfer function ( $\tan s i g$ ) from input layer to hidden layer, and Log-Sigmoid transfer function ( $\log s i g$ ) from hidden layer to output layer. Where $\tan \operatorname{sig}(n)=\frac{1-e^{-2 x}}{1+e^{-2 x}}$, $\log \operatorname{sig}(n)=\log \frac{1}{1+e^{-x}}$ 
To facilitate the neural network learning, we extract the first 300 samples to form the input vector matrix of the input layer neural network learning sample and the expected value matrix of learning sample.

Where each row of matrix A contains 300 companies' data, each column consists of standardized value of nine indicators. And in matrix B, the row No. located with 1 in each column means the corresponding company's clustering result

In this paper, the neural network training and analysis are realized by Matlab 7.1. After 189 times training of sample data, it reaches a certain steady state and the network arrives convergent. In the output matrix, those locations marked with 1 in each column represent the financial condition of corresponding sample. In the output matrix, each value of elements fit well when the weights are determined. As the mapping rule extraction completed, the early warning model is established.

Take companies No.301 to 447 as samples for test analysis to form an input vector matrix C and the expected value matrix D of test sample.

Use the weight matrix and threshold matrix gained by learning and training in test sample simulation, we could check up the correctness and accuracy of the model. Input these sample to the ready neural network model, after Matlab processing, we get the final output value matrix M.

Compared with the expected value matrix D of test sample, the classification discriminant of sample companies are briefly showed in Table 2 .

Table 2 Comparing results

\begin{tabular}{c|ccc}
\hline & Sample amount & Error amount & Correctness \% \\
\hline Healthy & 18 & 0 & 100 \\
Good & 29 & 1 & 96.6 \\
Moderate & 34 & 1 & 97.1 \\
Inferior & 34 & 0 & 100 \\
Warning & 32 & 0 & 100 \\
Total & 147 & 2 & 98.6 \\
\hline
\end{tabular}

As shown above, the correct rate of prediction using BP neural network simulation constructed by training method presents $98.6 \%$. Among those data, one in the Good level and one in the Moderate level are determined error. Generally the prediction is well and the network is effective. This model could be used in financial prediction of manufacturing industry. After integrating financial data of target companies into input vector, the output results would correspondingly determine the financial condition. And according to the outputs, those companies classified as Inferior have the possibility of further deterioration. While those classified as Warning should be a focus in case of financial distress.

\section{Conclusion}

This paper has provided an exposition of rough set discernibility matrix based on attributes reduction and improved neural network model. By introducing the hierarchical clustering analysis, the neural network model was designed with reasonable and accurate nodes number of output layer. And the breakthrough of healthy \& bankrupt classification could provide a multilevel warning system that is more precise. Thus, combined with the network topology of neural network analysis, a new warning rule would be developed.

Compared to the classical model of financial prediction, the use of discernibility matrix attribute reduction and five-level cluster analysis were found significant to maximize the avail of neural network model. Therefore, the input samples and output targets are optimized, as well as the predictive power of the early-warning model. 


\section{Reference}

[1]. Lee K., Booth D., Alam P. A comparison of supervised and unsupervised neural networks in predicting bankruptcy of Korean firms [J]. Expert Systems with applications. 29(2005): 1-16.

[2]. Min S., Lee J., Han I. Hybrid genetic algorithms and support vector machines for bankruptcy prediction [J]. Expert Systems with Applications. 31(2006).:652-660.

[3]. Cho S., Kim J., Bae J.K.. An integrative model with subject weight based on neural network learning for bankruptcy prediction [J]. Expert Systems with Applications. 36(2009). 403-410.

[4]. Andrés J.D., Lorca P., Bankruptcy forecasting: A hybrid approach using Fuzzy c-means clustering and Multivariate Adaptive Regression Splines (MARS) [J]. Expert Systems with Applications. 38(2011).1866-1875.

[5]. I.M. Premachandra, Y. Chen, J. Watson. DEA as a tool for predicting corporate failure and success: A case of bankruptcy assessment [J].Omega. 39 (2011).620-626. 